\title{
Anatomopathology of the Superstructure of the Stapes in Patients with Otosclerosis
}

\author{
Bettina Carvalho ${ }^{1}$ Rogerio Hamerschmidt ${ }^{1}$ Jose Ederaldo Telles ${ }^{2}$ Nicole Richter ${ }^{3}$ \\ ${ }^{1}$ Department of ENT, IPO, Curitiba, PR, Brazil \\ ${ }^{2}$ Department of Pathology, HC/UFPR, Curitiba, PR, Brazil \\ ${ }^{3}$ Department of Pathology, IPO, Curitiba, PR, Brazil \\ Address for correspondence Rogerio Hamerschmidt, PhD, \\ Department of Pathology, HC/UFPR, Rua General Carneiro, 181 - Alto \\ da Glória, Curitiba, 80060-900, PR, Brazil \\ Int Arch Otorhinolaryngol 2015;19:1-4. \\ (e-mail: rogeriohamer@hotmail.com).
}

\begin{abstract}
Introduction Otosclerosis is a disease that causes bone resorption and deposition in the auditory structures, leading to deafness. Many studies have evaluated the histopathology of the stapes footplate in this disease (osteoblasts, osteoclasts, vascular proliferation, fibroblasts, and histiocytes), but we found no studies in the literature involving the histology of the superstructure of the stapes.

Objectives To perform an analysis under optical microscopy of histopathologic findings of the superstructure of the stapes from patients with otosclerosis.

Methods A contemporary cross-sectional cohort study of pathology analysis of superstructures of the stapes of patients with otosclerosis.

Results Fifteen superstructures of stapes in patients with otosclerosis operated in our service and four stapes of cadavers used for dissection (controls) were evaluated. No areas of bone resorption or deposition or presence of osteoclasts and osteoblasts in the superstructure of the stapes were found. However, we found in the more distal portions of the crura areas with prominent cementitious lines and woven bone, which was

Keywords

- otosclerosis

- deafness

- stapes

different than the mature trabecular bone found in the head of the stapes or in the controls.

Conclusion There were histologic changes in the superstructure of the stapes in patients with otosclerosis operated in our service.
\end{abstract}

\section{Introduction}

Otosclerosis is an inflammatory disease that causes bone resorption and deposition in the auditory structures, leading to conductive and/or sensorineural progressive deafness. Its etiology is still unknown, and there are only theories to explain it. ${ }^{1}$ Histologic alterations of otosclerosis have been well described and are usually found in the pericochlear region, oval window, and stapes base. These alterations include areas of increased cellularity and bone resorption and deposition (presence of osteoclasts and osteoblasts), increased vascularization, fibrous thickening of the mucosa, and tissue affinity for acid dyes. ${ }^{1,2}$ These alterations may coexist, so there are three histopathologic patterns: spongi-

received

February 13, 2014

accepted

March 28, 2014

published online

July 18, 2014 otic (cellular), sclerotic (dense mineralized bone), and mixed, because the disease is dynamic. ${ }^{3}$

Many studies have been conducted analyzing the histologic alterations of the footplate of the stapes in otosclerosis, but there is no histologic descriptions of its superstructure (crura and head) in the literature, although there are reports of otosclerosis in the ossicles. ${ }^{4}$

During surgery for otosclerosis, the stapedotomy, the stapes superstructure is removed and is replaced by a prosthesis. The objective of this work is to analyze under optical microscopy the histopathology of the stapes superstructure removed in surgery to assess whether there are any histologic changes caused directly or indirectly by otosclerosis. 
Table 1 Groups for patients with otosclerosis (OTS) and controls stapes: representativeness of the parts of the stapes superstructures

\begin{tabular}{|l|l|l|l|}
\hline \multirow{2}{*}{} & \multicolumn{3}{|l|}{ Representativeness } \\
\cline { 2 - 4 } & Head & Anterior Crus & Posterior Crus \\
\hline OTS $(n=15)$ & 15 & 11 & 8 \\
\hline Control $(n=4)$ & 4 & 4 & 2 \\
\hline
\end{tabular}

\section{Methods}

This study was approved by the Ethics Committee on Human Research of our hospital, under CAAE: 13158813.9.0000.5529. This is a study of a contemporary cross-sectional cohort, by histopathologic analysis of the superstructure of the stapes.

Included in the group of patients were adults ( $>18$ years) diagnosed with otosclerosis and with surgical indication, who underwent stapes surgery in our hospital, with removal of the stapes superstructure, which was sent for histopathologic analysis. The control group included stapes removed from cadavers used for dissection. Patients who had different diagnosis during surgery, did not undergo surgery, or did not obtain enough material for histopathologic examination were excluded.

The specimens removed at surgery were immediately fixed in formalin solution (10\%, buffered). After 24 hours of fixation, the specimens were subjected to decalcifying by formic acid (4\%) for 12 hours. They were then automatically processed, embedded in paraffin, and cut with a rotational microtome, with a thickness of $3 \mu \mathrm{m}$, and stained with hematoxylin and eosin. The analysis was performed under optical microscopy (with biological microscope Bio3 brand model BEL Photonics, Osasco, Brazil) coupled to Moticam 580 camera (Motic Asia, Hong Kong, China), which allows the transmission of high-resolution image directly to a computer and obtains photos directly through the software Motic Images Plus 2.0 (Motic Asia, Hong Kong, China).

The specimens were analyzed by the pathologist, who was blind to their origin (cases or controls). The analysis was divided between findings of the head, crura, and base (when present).

\section{Results}

Between March and August 2013, 17 anatomopathological examinations of the superstructure of the stapes removed in stapedotomy were performed; of these, 2 were excluded due to insufficient sample for pathologic examination, leaving 15 samples studied. Simultaneously, 4 stapes of cadavers used for dissection of the general population, age 20 to 50 years, were examined as controls.

Compared with the samples from the four controls, we observed subtle changes in the stapes superstructures of the 15 patients with otosclerosis, represented by apparent reduction in the thickness of the cartilaginous cap top and slight evidence of remodeled bone, newly formed at the level of crura, represented by prominent cementitious lines and woven bone. The other classic pathologic findings described in otosclerosis were absent. The results are shown in - Tables 1 and $\mathbf{2}$ and -Figs. 1 and 2.

\section{Discussion}

Otosclerosis is a localized disease of the metabolism of endochondral bone of the otic capsule (labyrinth), characterized by disordered resorption and subsequent bone deposition. ${ }^{5}$ The onset of the pathologic process takes place with the calcification of the annular ligament of the oval window, and by extension to the stapes footplate, causing its attachment, most commonly in the anterior portion, and then to the ossicular chain, leading to deafness. ${ }^{2}$ Anatomical sites of predilection of the otosclerosis are: (1) anterior to the oval window, (2) margins of the round window, and (3) apicalmedial wall of the cochlea. ${ }^{6}$ The superstructure of the stapes is not considered involved in the process of otosclerosis, as it is not part of the otic capsule due to its embryologic origin in the second branchial arch. Although numerous studies have examined the morphologic characteristics of otosclerotic

Table 2 OTS group: Summary of histological alterations

\begin{tabular}{|c|c|c|c|}
\hline Types of alterations & Categories & - & + \\
\hline \multirow[t]{6}{*}{ Otosclerosis active lesions } & Nonosseous hypercellular tissue & 0 & \\
\hline & Bone resorption/bone deposition activity & & \\
\hline & Osteoblasts/osteoclasts & & 1 \\
\hline & Prominent cementitious lines; woven bone & & $9 / 5$ \\
\hline & Mucous vasculofibrous increment & & 1 \\
\hline & Acidophilia of bone tissue & 0 & \\
\hline \multirow[t]{2}{*}{ Other classic criteria associated with otosclerosis } & Perivascular blue & 0 & \\
\hline & Central sponginosis and peripheral sclerosis (preotosclerosis) & 0 & \\
\hline \multirow[t]{3}{*}{ General } & Irregularities of the contours of the crura & & 1 \\
\hline & Reduction of the cartilaginous superior cap & & 9 \\
\hline & Fibrous ligament & & 7 \\
\hline
\end{tabular}

Abbreviations: -, absent; +, present; OTS, group with otosclerosis. 


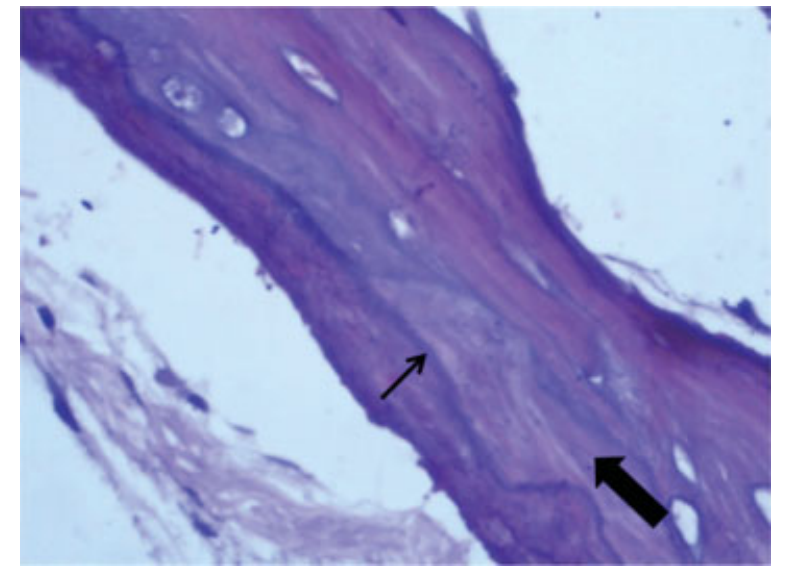

Fig. 1 Distal portion of the anterior crus of the stapes superstructure of a patient with otosclerosis showing dense cementitious line (thin arrow) and woven bone (thick arrow).

lesions, there are still limited studies about the alterations in the stapes. The existing studies mainly focus on the footplate but not the superstructure (crura and head). ${ }^{3}$ We sought, therefore, to evaluate the morphology and histopathology, under light microscopy, of the superstructures of the stapes removed during surgery for stapedectomy for otosclerosis performed in our department, to discover if there is any change in them at a histological level directly or indirectly caused by the disease.

Histopathology of otosclerosis is characterized by areas with presence of osteoclasts with bone destruction, osteoblasts, vascular proliferation, mucosal thickening, and presence of fibroblasts and histiocytes. New bone formation in the tissue increases the affinity for acid dyes, making it darker. ${ }^{2}$

No area of bone resorption or deposition or presence of osteoclasts and osteoblasts was found. However, in the more distal portions of the crura, areas of immature bone (woven bone) and prominent cementitious lines were found (-Fig. 1). One specimen showed the presence of numerous histiocytes in the mucosal epithelium lining the bone.

The cementitious lines are signs of osteogenesis or bone regeneration. They mark the boundary between the old portion of the trabecular bone, consisting of lamellar bone, and new bone, newly deposited by osteoblasts (wavy). The alterations of the cartilaginous cap are similar to the ones found in arthrosis of the joints. We do not know if these alterations of the crura are signs of otosclerosis of the superstructure or if they are an extension of the otosclerosis of the footplate (because we did not remove nor analyze the footplates on this sample), if the alterations represent another type of reaction of the bone of the crura to the presence of otosclerosis of the footplate (because the bone would be subjected to trauma by vibrating against a fixated footplate), or if the alterations are just an accidental finding (because they were not uniform in the studied material), but it is confirmed that there was histopathologic alterations in the superstructure of the stapes in otosclerosis.

Despite being a small sample size, this finding has not been described in the literature before; these were not obliterative otosclerosis (spreading of the otosclerosis foci beyond the oval window). We cannot say as well if these changes interfered with the outcome of the procedure, affected the prognosis of the disease, or led to any change in the audiometry, because the existing studies only analyzed the histology of the stapes footplate and not their crura. Thus, more studies are needed to confirm these findings and to elucidate these issues.

D'Alessandro et al studied the detailed morphology of the ossicles for the purpose of assisting the creation of prostheses. ${ }^{7}$ They found the presence of compact bone evident in the head, crura, and stapes footplate. Areas of cartilage were found in the top of the head and along the footplate. A thin epithelium could be seen covering the ossicles at its periphery, as we can observe in the bone controls (-Fig. 2).

In the histopathologic diagnosis, it is important to differentiate from other disorders of ossification. Osteogenesis can present similar to otosclerosis lesions, but some patients may present lesions with decreased calcification and microfractures. Paget disease is also similar to otosclerosis lesions, but with a different distribution, as it may affect other bones of the skeleton with a predilection for the axial skeleton, which is polyostotic in $70 \%$ of cases. Paget disease can cause deafness, tinnitus, or vertigo by affecting the bony labyrinth. It has the characteristic to affect an

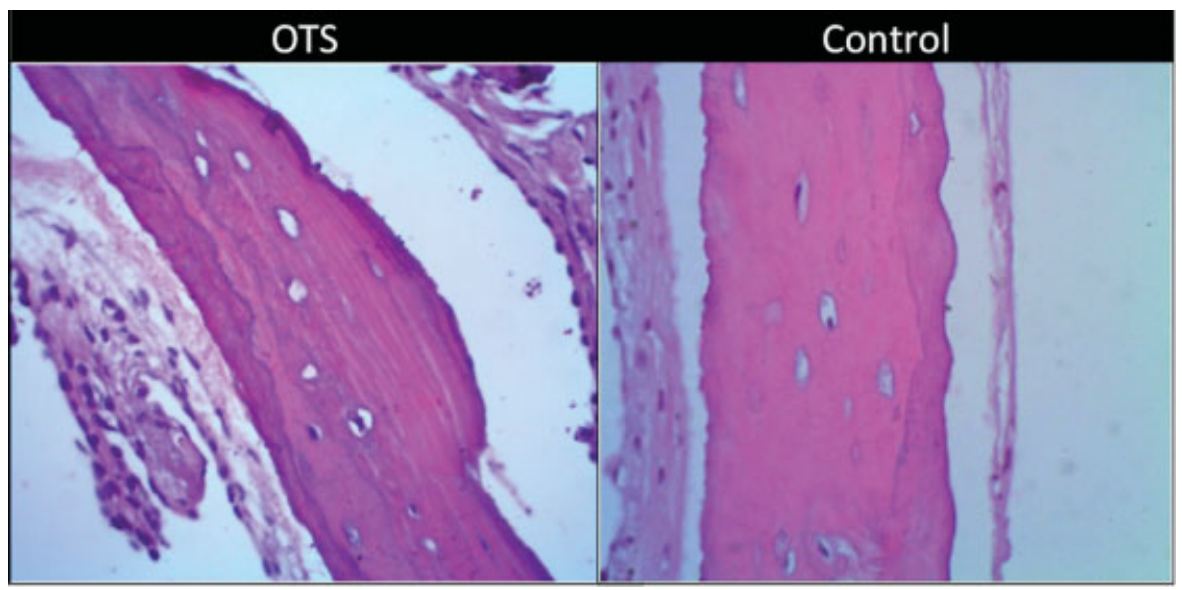

Fig. 2 Comparison between stapes superstructure (OTS) in patients with otosclerosis and control (CONTROL). 
older population than otosclerosis, and it affects males more than females. Histology shows multinucleated osteoclasts and cuboid osteoblasts. ${ }^{5,8}$

The evaluation of histopathologic findings described in this study can be enhanced with:

- electron microscopy;

- morphometry technique of the cartilaginous cap;

- basic immunohistochemistry to identify osteoblasts, osteoclasts, macrophages;

- expansion of the sample to achieve adequate sample size.

We also look forward to studies to evaluate whether the histopathologic alterations found here correlate with findings during surgery and postoperative audiometric evaluation.

\section{Conclusion}

Histologic alterations were found in the superstructure of the stapes of patients with otosclerosis operated in our service compared with controls.

\section{References}

1 Thomas JP, Minovi A, Dazert S. Current aspects of etiology, diagnosis and therapy of otosclerosis. Otolaryngol Pol 2011; 65(3):162-170

2 Cureoglu S, Schachern PA, Ferlito A, Rinaldo A, Tsuprun V, Paparella MM. Otosclerosis: etiopathogenesis and histopathology. Am J Otolaryngol 2006;27(5):334-340

3 Djeric D. Stapes pathology in otosclerosis: scanning electron microscopic examination. Adv Otorhinolaryngol 2007; 65:59-60

4 Escada PA, Capucho C, Chorão M, da Silva JF. Otosclerosis of the incus. Otol Neurotol 2007;28(3):301-303

5 Chole RA, McKenna M. Pathophysiology of otosclerosis. Otol Neurotol 2001;22(2):249-257

6 Schuknecht HF, Barber W. Histologic variants in otosclerosis. Laryngoscope 1985;95(11):1307-1317

7 D’Alessandro D, Danti S, De Vito A, Forli F, Bruschini L, Berrettini S. Histologic characterization of human ear ossicles for the development of tissue-engineered replacements. Otol Neurotol 2012; 33(8):1458-1468

8 Seitz S, Priemel M, Zustin J, et al. Paget's disease of bone: histologic analysis of 754 patients. J Bone Miner Res 2009; 24(1):62-69 